Pacific Journal of Mathematics

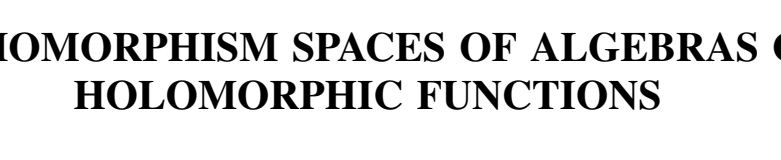




\title{
HOMOMORPHISM SPACES OF ALGEBRAS OF HOLOMORPHIC FUNCTIONS
}

\author{
P. J. DE PAEPE
}

\begin{abstract}
In this paper rationally convex sets, holomorphically convex sets and holomorphic sets in $\mathrm{C}^{n}$ are under consideration. Topological properties of these sets are given and attention is paid to examples showing that these three concepts are different. In the second section of the paper an example is given of a fat, connected, holomorphically convex set, which is not a holomorphic set.
\end{abstract}

A compact subset $K$ of $\mathbf{C}^{n}$ is called rationally convex if $K$ can be identified with the set of continuous complex-valued nontrivial homomorphisms of the function algebra $R(K)$, i.e. the algebra of uniform limits on $K$ of restrictions to $K$ of rational functions whose pole sets miss $K$. So $K$ is rationally convex if and only if the rationally convex hull $r(K)$ of $K$ defined by

$$
\begin{aligned}
r(K)= & \left\{x \in \mathbf{C}^{n}:|r(x)| \leqq\|r\|_{K} \text { for all rational functions } r\right. \\
& \text { not having poles on } K\} \\
= & \left\{x \in \mathbf{C}^{n}: p(x) \in p(K) \text { for every polynomial } p\right\}
\end{aligned}
$$

equals $K$. As usual $\|\cdot\|_{K}$ denotes the supremum norm on $K$. A compact set $K$ in $\mathbf{C}^{n}$ is holomorphically convex if it is the space of continuous complex-valued nontrivial homomorphisms of $H(K)$, the function algebra on $K$ consisting of uniform limits on $K$ of restrictions to $K$ of functions holomorphic in a neighborhood of $K . \quad K$ is said to be a holomorphic set if $K$ is a countable intersection of Stein manifolds in $\mathbf{C}^{n}$. We recall that a Stein manifold of dimension $n$ is a complex analytic manifold such that the following three conditions are satisfied: $\operatorname{Hol}(M)$, the collection of holomorphic functions on $M$, separates the points of $M$; for every $x \in M$ there exist $f_{1}, \cdots, f_{n} \in \operatorname{Hol}(M)$ such that $f_{1}, \cdots, f_{n}$ provide local coordinates at $x ; \operatorname{Hol}(M)-\operatorname{hull}(K)=\{x \in M:|f(x)| \leqq$ $\|f\|_{K}$ for all $\left.f \in \operatorname{Hol}(M)\right\}$ is a compact subset of $M$ for every compact subset $K$ of $M$. Note that an open subset of $\mathbf{C}^{n}$ is Stein if the last condition is satisfied. Of primary importance is the property of Stein sets $M \subset \mathbf{C}^{n}$, that the continuous homomorphism space of $\operatorname{Hol}(M)$ equals $M$. Using this fact and a characterization of holomorphically convex subsets of $\mathbf{C}^{n}$ of Birtel, [2], it is evident that holomorphic sets are holomorphically convex. 
If $K$ is a compact subset of $\mathbf{C}^{n}, r(K), h c(K), h s(K)$ respectively denote the rationally convex hull of $K$, the smallest holomorphically convex set in $\mathbf{C}^{n}$ containing $K$, and the smallest holomorphic set in $\mathbf{C}^{n}$ containing $K$, the existence of which is shown in [11], chapter III.

Let $M$ be a complex analytic manifold. $\operatorname{Mer}(M)$ will be the collection of meromorphic functions on $M$, i.e. "functions" which locally are the quotient of two holomorphic functions, the denominator not identically zero on a component of its domain of definition. If $M$ is Stein it can be shown that every meromorphic function on $M$ is the quotient of two elements of $\operatorname{Hol}(M)$. If $K$ is a compact subset of $M, \operatorname{Mer}(M)-$ hull $(K)$ will denote the set of points in $M$ at which $|m(x)| \leqq\|m\|_{K}$ for all $m \in \operatorname{Mer}(M), m$ holomorphic near $K$.

We also need Birtel's characterization of holomorphically convex sets in $\mathbf{C}^{n}$, [2]. Let $K$ be a compact subset of $\mathbf{C}^{n}$. Let $U$ be an open neighborhood of $K$ and let $E(U)$ be the continuous homomorphism space of $\operatorname{Hol}(U)$. By a famous theorem of Bishop $E(U)$ can be given the structure of a Stein manifold in such a way that $\operatorname{Hol}(U)$ is isomorphic to $\operatorname{Hol}(E(U))$, the isomorphism given by $f \rightarrow \hat{f}$ where $\hat{f}(\phi)=\phi(f)$ for all $\phi \in E(U)$. Note that $E(U)$ coincides with $U$, if $U$ is Stein. Let $\Pi: E(U) \rightarrow \mathbf{C}^{n}$ be the map

$$
\Pi(\phi)=\left(\phi\left(Z_{1}\right), \cdots, \phi\left(Z_{n}\right)\right)=\left(\hat{Z}_{1}(\phi), \cdots, \hat{Z}_{n}(\phi)\right)
$$

$Z_{l}$ being the $i$ 'th coordinate function on $\mathbf{C}^{n}$. Then $K$ is holomorphically convex if and only if $K=\cap\{\Pi E(U): U \supset K\}$.

For general background information about function algebras, complex analytic manifolds and complex analytic varieties we refer to [4] and [5].

1. Topological properties of homomorphism spaces. Let $K$ be a compact subset of $\mathbf{C}^{n}$ and let $Y$ be a compact holomorphically convex set in $\mathbf{C}^{n}$, containing $K$. We define $M(Y, K)$ as the set of all points $x \in Y$ with the property that for all compact subsets $S$ of $Y$, containing $x$, for all open neighborhoods $U$ (in $\mathbf{C}^{n}$ ) of $S$ and for all $f \in \operatorname{Hol}(U)$ with $f(x)=0, f$ attains the value zero on $\operatorname{bdr} S \cup$ $(S \cap K)$. Here bdr $S$ denotes the boundary of $S$ relative to $Y$. Note that $K \subset M(Y, K) \subset Y$.

\section{THEOREM 1. $M(Y, K)$ is holomorphically convex.}

Proof. Suppose the theorem is false, then $A=\cap\{\Pi E(U), U$ open in $\left.\mathbf{C}^{n}, U \supset M(Y, K)\right\}$ is a subset of $Y$ and $A$ properly contains $M(Y, K)$. Let $x \in A, x \notin M(Y, K)$. There exist $S \subset Y$, with $x \in S$, a neighborhood $U$ of $S$ and a function $f \in \operatorname{Hol}(U)$ such that $f(x)=$ 
$0 \notin f($ bdr $S \cup(S \cap K)$. By definition of $M(Y, K), f$ has no zeroes on $M(Y, K) \cap S$. Let $Z$ be the zero set of $f$. By shrinking $U$, if necessary, we may suppose $Z$ has a positive distance to $Y \backslash S$, in particular $Z \cap Y=Z \cap S$. Let $U^{\prime}$ be a neighborhood of $S$, relatively compact in $U$. Let $W$ be a neighborhood of $M(Y, K)$ such that $\Pi E(W) \cap Z$ is contained in $U^{\prime} \cap \Pi E(W)$. Clearly $\Pi E(W \backslash Z) \subset \Pi E(W)$. We show $\Pi E(W \backslash Z) \subset(\Pi E(W)) \backslash Z$. Suppose this is false, let

$$
\begin{array}{ll}
E_{1}=\Pi^{-1}(U \cap \Pi E(W \backslash Z)) \cap E(W \backslash Z), & h_{1}=1 / f \circ \Pi, \\
E_{2}=E(W \backslash Z) \backslash \operatorname{cl}\left(\Pi^{-1}\left(\Pi E(W \backslash Z) \cap U^{\prime}\right)\right), & h_{2}=0 .
\end{array}
$$

Here cl stands for closure. Then, because Cousin I problems are solvable on the Stein manifold $E(W \backslash Z)$ ([5], p. 248), there is a meromorphic function $m$ on $E(W \backslash Z)$ such that $m-1 / f \circ \Pi$ is holomorphic on $E_{1}$ and $m$ is holomorphic on $E_{2}$, so $m$ is holomorphic on $W \backslash Z$. Therefore $m$ is in $\operatorname{Hol}(E(W \backslash Z))$, contradicting the definition of $m$. So we have shown $\{y \in E(W \backslash Z)$ : $\Pi(y) \in Z\}$ is empty.

It follows that $W \backslash Z \supset M(Y, K), x \notin \Pi E(W \backslash Z)$, hence $x \notin A$, in contradiction with our initial assumption. Therefore $M(Y, K)$ is holomorphically convex.

THEOREM 2. If $Y$ is a holomorphic set, then $M(Y, K)$ is a holomorphic set.

Proof. Let $x \in Y \backslash M(Y, K)$. As in the proof of the previous theorem, let $S, U, f, Z$ and $U^{\prime}$ be given. Choose a Stein manifold $W$ containing $Y$ such that $Z \cap W$ is contained in $U^{\prime} \cap W$. Solving a Cousin I problem on $W$ we find a meromorphic function $m$ on $W$ such that $m-1 / f$ is holomorphic on $U \cap W$ and $m$ is holomorphic on $W \backslash \operatorname{cl}\left(U^{\prime}\right)$. Then the Stein manifold $\{y \in W:|m(y)|<C\}$ for $C$ sufficiently large does not contain $x$ and contains $M(Y, K)$. Thus $M(Y, K)$ is the intersection of all Stein manifolds containing $M(Y, K)$, so $M(Y, K)$ is a holomorphic set.

In the case $Y$ is a holomorphic set which has a trivial second cohomology group with coefficients in the integers, we have the following characterization of $M(Y, K)$ :

THEOREM 3. Let $Y$ be a holomorphic set and suppose $H^{2}(Y ; \mathbf{Z})$ $=0$. Then $M(Y, K)=\cap\left\{\operatorname{Mer}(U)-\operatorname{hull}(K)\right.$ : $U$ open in $\left.\mathbf{C}^{n}, U \supset Y\right\}$.

Proof. Let $x \in Y, x \notin \cap\{\operatorname{Mer}(U)-\operatorname{hull}(K): U \supset Y\}$. Then there are a Stein manifold $U$ containing $Y$ and $m \in \operatorname{Mer}(U)$ such that $m$ is holomorphic near $K$ and $m(x)=1>\|m\|_{K}$. By shrinking $U$, if necessary, we may assume that there are $f, g \in \operatorname{Hol}(U)$, relatively prime such 
that $m=f / g$ (see [5], p. 251). Now the analytic variety $W=$ $\{y \in U: f(y)=g(y)\}$ does not meet $K$ because at every point in $K \cap W$ the irreducible branches of $W$ through this point are contained in $\{y \in U: f(y)=g(y)=0\}$ since $f / g$ is holomorphic near $K$. This would contradict the fact that $f$ and $g$ are relatively prime. So $h=f-g$ has a zero at $x$ and does not attain the value zero on $K$. Let $\varepsilon>0$ be such that $|h(y)|>\varepsilon$ for all $y \in K$, then $S=\{y \in Y:|h(y)| \leqq \varepsilon\}$ is a compact subset of $Y, x \in S, K \cap S=\varnothing$ and bdr $S \subset\{y \in Y:|h(y)|=\varepsilon\}$. Hence $x \notin M(Y, K)$.

Conversely, suppose $x \notin M(Y, K)$, so there are a subset $S$ of $Y$, $x \in S$, a neighborhood $U$ of $S$ and $f \in \operatorname{Hol}(U)$ such that $f(x)=0$ and $f$ has no zeroes on bdr $S \cup(S \cap K)$. Again we may assume that the only zeroes of $f$ in $Y$ are in $S$. Let $W$ be a Stein manifold containing $Y$ such that the zero set $Z$ of $f$ in $U$ is an analytic hypersurface in $W$. Without loss of generality we may assume there exists $h \in \operatorname{Hol}(W)$ such that $Z=\{y \in W: h(y)=0\}$ (see [9], p. 286). Hence $h(x)=0$ and $h$ does not attain the value zero on $K$ (even not on $M(Y, K)$ ), so $|h(y)|>2 \varepsilon$ for all $y \in K$ and some $\varepsilon>0$. Hence $|(1 / h+\varepsilon)(x)|>\|1 / h+\varepsilon\|_{K}$, so $x \notin \operatorname{Mer}(W)-\operatorname{hull}(K)$.

If we restrict ourselves to rationally convex sets $Y$, we obtain the following result:

THEOREM 4. Let $Y$ be rationally convex and $H^{2}(Y ; Z)=0$. Then $M(Y, K)$ is rationally convex.

Proof. As in the proof of Theorem 3, if $x \in Y \backslash M(Y, K)$, then there exists a Stein manifold $W, W \supset Y$, and $h \in \operatorname{Hol}(W)$ such that $h(x)=$ $0 \notin h(M(Y, K))$. Because $Y$ is rationally convex, $h$ can be approximated on $Y$ by rational functions with pole sets which miss $Y$. So there is a rational function $r$, holomorphic near $Y$ with $0<|r(x)|<|r(y)|$ for all $y \in M(Y, K)$. So $|1 / r(x)|>\|1 / r\|_{M(Y, K)}$, hence

$$
x \notin r(M(Y, K)) \text {. }
$$

Hence $M(Y, K)$ is rationally convex.

The next lemma has important consequences.

Lemma 1. Let $x \in Y \backslash K$. If there exists a neighbourhood $U$ of $x$ and a one-dimensional analytic subvariety $V$ of $U$ such that $Y \cap U \subset V$ then' $x \notin M(Y, K)$.

Proof. Suppose such $U$ and $V$ do exist. We may assume $U \cap K=$ $\varnothing$. Let $f$ be a holomorphic function in a neighborhood $W \subset U$ of $x$ such that $f(x)=0$ and $f(y) \neq 0$ if $y \in V \cap W, y \neq x$. Let $S$ be a compact subset 
of $Y$ containing $x$ in its interior and such that $S \subset W$. Then $S \cap K=\varnothing$ and $0 \notin f(\operatorname{bdr} S)$. Hence $x \notin M(Y, K)$.

THEOREM 5. The sets $h c(K), h s(K)$ do not contain points $x \notin K$ such that there are a neighborhood $U$ of $x$ and a onedimensional analytic subvariety $V$ of $U$ with the property $h c(K) \cap U \subset V$, respectively $h s(K) \cap$ $U \subset V$. The same statement holds for $r(K)$ if $H^{2}(r(K) ; Z)=0$.

Proof. Note that $h c(K)=M(h c(K), K), h s(K)=M(h s(K), K)$ and $r(K)=M(r(K), K)$ if $H^{2}(r(K) ; \mathbf{Z})=0$. Now apply Lemma 1 .

In the literature appear at least three examples of holomorphic sets which are not rationally convex. First, Wermer in [10] gives an example of a set $K$ which is the biholomorphic image of a polydisc in $\mathbf{C}^{2}$ which is not polynomially convex:

$K=\left\{\left(z_{1}, z_{2}, z_{3}\right) \in \mathbf{C}^{3}: z_{1}=z, z_{2}=z w, z_{3}=w(z w-1),|z| \leqq 1,|w| \leqq 1\right\}$. Stolzenberg later in [8] shows that $K$ is even not rationally convex and $r(K)=K \cup D$, where

$$
D=\left\{\left(z_{1}, z_{2}, z_{3}\right):\left|z_{1}\right|<1, z_{2}=1, z_{3}=0\right\} .
$$

Lemma 1 shows that $K$ is a holomorphic set and $M(r(K), K)=K$; from Theorem 4 it follows that $H^{2}(r(K) ; \mathbf{Z})$ is nontrivial. Stolzenberg in [9], p. 272, gives an example of two disjoint polynomially convex sets $K_{1}$ and $K_{2}$ whose union $K$ is not polynomially convex.

$$
\begin{aligned}
& K_{1}=\left\{\left(z_{1}, z_{2}, z_{3}\right) \in \mathbf{C}^{3}:\left(z_{3}-1\right)\left(z_{1} z_{2}-1\right)=0,\left|z_{3}\right| \leqq 1,\left|z_{1}\right| \leqq 2,\left|z_{2}\right| \leqq \frac{1}{2}\right\} \\
& K_{2}=\left\{\left(z_{1}, z_{2}, z_{3}\right) \in \mathbf{C}^{3}:\left|z_{1}\right| \leqq 1,\left|z_{2}\right| \leqq 1, z_{3}=0\right\} \\
& K=K_{1} \cup K_{2} .
\end{aligned}
$$

Stolzenberg shows that $r(K) \supset S=\left\{\left(z_{1}, z_{2}, z_{3}\right): z_{1} z_{2}=1, z_{3}=0,1 \leqq\right.$ $\left.\left|z_{1}\right| \leqq 2\right\}$. Now it is easily seen that $r(K)=K \cup S$. Now bdr $S \cup(S \cap K)=$ bdr $S=S \cap K$. Therefore, for $x=\left(x_{1}, x_{2}, x_{3}\right) \in S$, $x \notin \operatorname{bdr} S, z_{1}-x_{1}$ attains the value zero at $x$ and $0 \notin\left(z_{1}-x_{1}\right)(\mathrm{bdr} S)$, hence $x \notin M(r(K), K)$. So $M(r(K), K)=K, K$ is a holomorphic set, and by Theorem $4, H^{2}(r(K) ; \mathbf{Z}) \neq 0$.

Much earlier Oka, [6], constructs a Stein manifold $M$ in $\mathbf{C}^{2}$ with the property that not every holomorphic function on $M$ can be approximated by rational functions on compact subsets of $M$. His domain $M$ is of the form

$R \cap\{|f|<A\}, \quad$ where 
$R=\left\{\left(z_{1}, z_{2}\right) \in \mathbf{C}^{2}: \mid r_{i}\left(z_{1}, z_{2}\right)<1, i=1, \cdots, N\right\}, \quad r_{i}$ rational functions for $i=1, \cdots, N$, and where $f$ is a meromorphic function on $R$, gotten in the following way. There is a polynomial $h$ whose zero set in $R$ consists of two components $V_{1}$ and $V_{2}$; now $f$ is the solution of a Cousin I problem on $R$, holomorphic near $V_{1}$ and such that $f-1 / h$ is holomorphic near $V_{2}$ (so $M \cap V_{2}=\varnothing$ ).

Oka constructs a compact set $F$ in $M$ such that $r(F) \neq F$, in fact $r(F) \cap V_{2} \neq \varnothing$. Now $K=\{x \in r(F):|f(x)| \leqq B\}$ is a holomorphic set containing $F$ if $B$ is sufficiently large and is not rationally convex. Let $S$ be the closure of $r(K) \backslash K$, then bdr $S \cup(S \cap K) \subset\{|f|=B\}$. By blowing up the functions $r_{i}$ a little and enlarging $A$ and $B$ if necessary, we may assume $\{|f|=B\} \cap V_{1}=\varnothing$. So the polynomial $h$ attains the value zero on $S$ and has no zeroes on $\{|f|=B\}$. Hence $M(r(K), K) \neq r(K)$, so by Theorem $4, H^{2}(r(K) ; \mathbf{Z}) \neq 0$.

It is apparent from the preceding discussion that all three examples have a topological property in common: the second cohomology group of the rationally convex hull of the set with coefficients in the integers does not vanish. One might wonder whether it is possible to put purely topological conditions on a holomorphic set or its rationally convex hull to ensure that the set is rationally convex. Problem:

If $K$ is a holomorphic set in $\mathbf{C}^{n}$ and if $H^{2}(r(K) ; \mathbf{Z})=0$, is $K$ then rationally convex?

\section{A fat connected holomorphically convex set, not a} holomorphic set. We will now give an example of a compact set $X$ in $\mathbf{C}^{3}$ which is holomorphically convex, but is not a holomorphic set. Moreover $X$ will be connected and fat, i.e. $X$ is the closure of its interior.

In [3] Björk gives the following example of a holomorphically convex set in $\mathbf{C}^{2}$, not a holomorphic set:

$$
\begin{aligned}
K= & \{(z, w): z w=0,|z| \leqq 1,|w| \leqq 1\} \\
& \cup \bigcup_{n=1}^{\infty}\left\{(z, w): 2^{-n-1} \leqq|z| \leqq 2^{-n},|w|=1-1 / n, n \in \mathbf{N}\right\} .
\end{aligned}
$$

He then imbeds this set in $\mathbf{C}^{3}$ and connects up the components by thin spiral-like sets in order to get a holomorphically convex set, not a holomorphic set, which is connected but has no interior ([3]).

Consider the following subsets of the complex $t$-plane:

$$
T_{n}=\left\{2^{-2 n-1} \leqq \arg t \leqq 2^{-2 n},|t| \leqq a_{n}\right\},
$$


where $n \in \mathrm{N}$ and $a_{n}, a_{n} \rightarrow 0$, are small positive numbers to be determined later.

$$
\begin{aligned}
T_{0} & =\left\{|t-1| \leqq 1, \operatorname{Im} t \leqq 0=\left\{|t-1| \leqq 1,\left|e^{-i t}\right| \leqq 1\right\}\right. \\
S_{n} & =\left\{\left|t+2^{-4 n-1}\right| \geqq 2^{-4 n-1},\left|t+2^{-4 n}\right| \leqq 2^{-4 n}\right\}, \quad n \in \mathbf{N} \\
T & =\bigcup_{n=0}^{\infty} T_{n} \cup \bigcup_{n=1}^{\infty} S_{n} .
\end{aligned}
$$

We need two properties of the algebra $R(T): t=0$ is a peak point for $R(T)$, i.e. there exists $f \in R(T)$ with $f(0)=1$ and $|f(t)|<1$ for all $t \in T, t \neq 0$. Furthermore for $t \in Q \backslash\{0\}$, where $Q$ is one of the sets $T_{n}$ or $S_{n}$, there exists $f \in R(T)$ with $f=0$ on $T \backslash Q$ and $f(t)=1$. This follows from the fact that every continuous function on $T$ which is analytic in the interior of $T$ can be approximated on $T$ by rational functions with poles not meeting $T$.

Now define subsets of $\mathbf{C}^{3}$ by

$$
\begin{aligned}
B_{n}= & \left\{(z, w, t): 2^{-n-1} \leqq|z| \leqq 2^{-n}, 1-2^{-2 n} \leqq|w|\right. \\
& \left.\leqq 1-2^{-2 n-1}, t \in T_{n}\right\}, \quad n \in \mathbf{N} \\
B_{0}= & \left\{(z, w, t):|z w \pm(1-t)| \leqq 1,\left|e^{-i t}\right| \leqq 1,|z| \leqq 1,|w| \leqq 1\right\} .
\end{aligned}
$$

It is easily seen that $B_{n}, n \geqq 0$, is connected and is the closure of its interior. Our desired set $X$ will be the union of the sets $B_{n}, n \geqq 0$, and sets $A_{n}, n \geqq 1$, where $A_{n}$ connects $B_{n}$ and $B_{n+1}$, and such that $A_{n} \cap$ $\{t=0\} \subset\left(B_{n} \cup B_{n+1}\right) \cap\{t=0\}$ and

$$
|z| \leqq 2,|w| \leqq 2, t \in S_{n} \quad \text { for }(z, w, t) \in A_{n} .
$$

Define the function $f_{n}$, analytic in a neighborhood of $X$ as the branch of $1 / 2 \pi i \log 2^{4 n+1}\left(t+2^{-4 n-1}\right)$ which is $-(n-1)$ on $B_{1} \cap\{t=0\}, \cdots,-1$ on $B_{n-1} \cap\{t=0\}, 0$ on $B_{n} \cap\{t=0\}$ and 1 on $\left(X \backslash\left(B_{1} \cup \cdots \cup B_{n}\right)\right) \cap\{t=0\}$. So, putting $\beta_{n}=2^{4 n+1}\left(t+2^{-4 n-1}\right), \quad f_{n}(t)=1 / 2 \pi i \log \left|\beta_{n}\right|+1 / 2 \pi \arg \beta_{n}$, where $\arg \beta_{n}$ ranges on $X$ from approximately $-(n-1) 2 \pi$ to approximately $2 \pi$.

Now on $B_{1} \cup A_{1} \cup \cdots \cup A_{n-2} \cup B_{n-1}, \quad \arg \beta_{n}<-3 \pi / 2$, hence $\operatorname{Re} 3 f_{n}<-9 / 4$. Similarly on $X \backslash\left(B_{1} \cup A_{1} \cup \cdots \cup B_{n} \cup A_{n}\right)$, $\operatorname{Re} 3 f_{n}>$ $9 / 4$. Since $|z| \leqq 2,|w| \leqq 2$ for $(z, w, t) \in X$, the zero sets of $i z-3 f_{n}$ and $i w-3 f_{n}$ do not meet $X \backslash\left(A_{n-1} \cup B_{n} \cup A_{n}\right)$.

Since $f_{n}=0$ on $B_{n} \cap\{t=0\}$ and $z$ and $w$ have no zeroes on $B_{n}$, we can make $a_{n}$ (see the definition of $T_{n}$ ) sufficiently small to assure that the zero sets of $i z-3 f_{n}$ and $i w-3 f_{n}$ do not meet $B_{n}$. We will construct the sets $A_{n}$ in such a way that the zero sets of $i z-3 f_{n}, i z-3 f_{n+1}$, $i w-3 f_{n}$, 
iw $-3 f_{n+1}$ do not meet $A_{n}$. This means that $g_{n}=1 /\left(i z-3 f_{n}\right)$ and $h_{n}=$ $1 /\left(i w-3 f_{n}\right)$ are holomorphic in a neighborhood of $X$, i.e. $h_{n}, g_{n} \in H(X)$.

We proceed with the construction of the sets $A_{n}$. Consider the following inequalities defining $A_{n}$.

$$
\begin{aligned}
& \left|t+2^{-4 n-1}\right| \geqq 2^{-4 n-1} \\
& \left|t+2^{-4 n}\right| \leqq 2^{-4 n} \\
& \left|2^{n+2}\left(z-2^{-n-1}\right) \pm\left(1-f_{n}\right)\right| \leqq 1 \\
& \left|2^{n+2}\left(z-2^{-n-1}\right) \pm f_{n}\right| \leqq 1 \\
& \left|2 n\left(w-\left(1-2^{-2 n-1}\right)\right) \pm\left(1-f_{n}\right)\right| \leqq 1 \\
& \left|2 n\left(w-\left(1-2^{-2 n-2}\right)\right) \pm f_{n}\right| \leqq 1
\end{aligned}
$$

From (3) and (5) it follows that $\operatorname{Re} z>0$ on $A_{n}$ since $\left|2^{n+2}\left(z-2^{-n-1}\right)\right| \leqq 1$ and $\left|2 n\left(w-\left(1-2^{-2 n-1}\right)\right)\right| \leqq 1$ on $A_{n}$. Also from the definition of $f_{n}$, $\operatorname{Im} f_{n} \leqq 0$ on $A_{n-1} \cup A_{n}, n \geqq 1, A_{0}:=\varnothing$, since $t \in S_{n}$ if $(\dot{z}, w, t) \in A_{n}$. So $i z-3 f_{n}$ and $i w-3 f_{n}$ cannot have zeroes on $A_{n-1} \cup A_{n}$, so $g_{n}$ and $h_{n}$ belong to $H(X)$. Now it is easily seen that

(i) $\quad A_{n}$ is connected

(ii) $A_{n}$ connects up $B_{n}$ and $B_{n+1}$ and $A_{n} \cap\{t=0\} \subset\left(B_{n} \cup B_{n+1}\right) \cap\{t=0\}$

(iii) $A_{n}$ is the closure of its interior

(iv) $A_{n}$ as a closed analytic polyhedron is holomorphically convex.

Define $X=\cup B_{n} \cup \cup A_{n}$. First we show that the space $\Delta H(X)$ of continuous complex-valued nontrivial homomorphisms of $H(X)$ can be indentified with $X$, i.e. $X$ is holomorphically convex.

Let $\phi \in \Delta H(X)$, so $\phi(t) \in T$.

Suppose $\phi(t)=0$. Since $t=0$ is a peak point of $R(T)$, there is a function $g \in R(T)$ peaking at $t=0$. Now $\phi \in \Delta[H(X) \mid X \cap\{t=0\}]$ where $[H(X) \mid X \cap\{t=0\}]$ is the function algebra on $X \cap\{t=0\}$ generated by restrictions of elements of $H(X)$. Suppose this is not the case, then there is $f \in H(X)$ with $\phi(f)=1$ and $1>\|f\|_{X \cap\{t=0\}}$. Consider $g$ in the natural way as an element of $H(X)$, then

$$
\phi\left(f g^{m}\right)=1>\left\|f g^{m}\right\|_{X}
$$

if $m$ is sufficiently large, in contradiction with $\phi \in \Delta H(X)$.

Let $m$ be a positive Jensen representing measure for $\phi$ on $X \cap$ $\{t=0\}$, representing $[H(X) \mid X \cap\{t=0\}]$. So 


$$
\log |\phi(f)| \leqq \int \log |f| d m
$$

and

$$
\phi(f)=\int f d m
$$

for $f \in[H(X) \mid X \cap\{t=0\}]$.

Suppose $m\left(B_{n} \cap\{t=0\}\right)>0$ for some $n \in \mathbf{N}$. There is a linear combination $F_{n}$ of the functions $f_{m}$ which is 1 on $B_{n} \cap\{t=0\}, 0$ on $\left(X \backslash B_{n}\right) \cap\{t=0\}$. Now

$$
\log \left|\phi\left(F_{n}-1\right)\right| \leqq \int \log \left|F_{n}-1\right| d m=-\infty .
$$

Hence $\phi\left(F_{n}\right)=1$ and it follows that $m\left(B_{n} \cap\{t=0\}\right)=1$, so $\phi \in$ $\Delta\left[H(X) \mid B_{n} \cap\{t=0\}\right]$. Now $i g_{n}$ and $i h_{n}$ are in $H(X)$ and restrict to $1 / z$ and $1 / w$ on $B_{n} \cap\{t=0\}$. So

$$
\left[H(X) \mid B_{n} \cap\{t=0\}\right]=R\left(B_{n} \cap\{t=0\}\right) .
$$

Since $\Delta R\left(B_{n} \cap\{t=0\}\right)=B_{n} \cap\{t=0\}, \phi \in B_{n} \cap\{t=0\}$.

If $m\left(B_{n} \cap\{t=0\}\right)=0$ for all $n \in \mathbf{N}, \quad m\left(B_{0} \cap\{t=0\}\right)=1$, so $\phi \in \Delta\left[H(X) \mid B_{0} \cap\{t=0\}\right.$. Since $B_{0} \cap\{t=0\}$ is polynomially convex, $\phi \in B_{0} \cap\{t=0\}$. So if $\phi \in \Delta H(X)$ and $\phi(t)=0, \phi \in X \cap\{t=0\}$. If $\phi(t) \in S_{n} \backslash\{0\}, \phi \in \Delta\left[H(X) \mid A_{n}\right]$. If not, there exists $g \in H(X)$ with $\phi(g)=1>\|g\| A_{n}$. But there is $f \in R(T)$ with $f(\phi(t))=1, f=0$ on $T \backslash S_{n}$. So $\phi\left(g^{m} f\right)=1>\left\|g^{m} f\right\|_{X}$ if $m$ is sufficiently large, a contradiction. Now $\Delta\left[H(X) \mid A_{n}\right]=A_{n}$ since the defining functions for $A_{n}$ are in $H(X)$. So $\phi \in A_{n}$.

If $\phi(t) \in T_{0} \backslash\{0\}, \phi \in \Delta\left[H(X) \mid B_{0}\right]$ and since $B_{0}$ is a compact closed analytic polyhedron in $\mathbf{C}^{3}$, defined by functions in $\operatorname{Hol}\left(\mathbf{C}^{3}\right)$

$$
\Delta\left[H(X) \mid B_{0}\right]=\Delta\left[\operatorname{Hol}\left(\mathbf{C}^{3}\right) \mid B_{0}\right]=B_{0},
$$

so $\phi \in B_{0}$. If $\phi(t) \in T_{n} \mid\{0\}, \phi \in \Delta\left[H(X) \mid B_{n}\right]$. Again we will show $\left[H(X) \mid B_{n}\right]=R\left(B_{n}\right)$. Now $g_{n}$ and $h_{n}$ are in $H(X)$. Making $a_{n}$, one of the determining constants of $B_{n}$ small enough to get

$$
\left|3 f_{n}\right|<\left|i z-3 f_{n}\right|, \quad\left|3 f_{n}\right|<\left|i w-3 f_{n}\right|
$$

on $B_{n}$, we have $1 / i z=\Sigma\left(-3 f_{n}\right)^{m} \cdot\left(g_{n}\right)^{m+1}$ where the series converges uniformly on $B_{n}$ to a function in $\left[H(X) \mid B_{n}\right]$. So $1 / z \in\left[H(X) \mid B_{n}\right]$ and similarly $1 / w \in\left[H(X) \mid B_{n}\right]$. So $\left[H(X) \mid B_{n}\right]=R\left(B_{n}\right)$ and hence $\phi \in B_{n}$. This shows $\Delta H(X)=X$. 
Now it is clear that $X$ is not a holomorphic set since $X \cap\{t=0\}$ is not a holomorphic set by Björk's example.

\section{REFERENCES}

1. F. T. Birtel, Function algebras and several complex variables, unpublished notes.

2. - Some holomorphic function algebras, Papers from the Summer Gathering on Function Algebras at Aarhus, July 1969, 11-18.

3. J. E. Björk, Holomorphic convexity and analytic structures in Banach algebras, Arkiv för Matematik, 9 (1971), 39-54.

4. T. Gamelin, Uniform Algebras, Prentice Hall Inc. 1969.

5. R. C. Gunning and H. Rossi, Analytic Functions of Several Complex Variables, Prentice-Hall Inc. 1965.

6. K. Oka, Sur les fonctions analytiques de plusieurs variables. IV-Domaines d'holomorphie et domaines rationellement convexes, Japan J. Math., 17 (1940), 517-521.

7. P. J. de Paepe, Analytic polyhedra, Tulane Dissertation, 1971.

8. G. Stolzenberg, An example concerning rational convexity, Math. Ann., 147 (1962), $275-276$.

9. G. Stolzenberg, Polynomially and rationally convex sets, Acta Math., 109 (1963), 259-289.

10. J. Wermer, An example concerning polynomial convexity, Math. Ann., 139 (1959), 147-150.

11. W. Zame, Stable algebras of holomorphic germs, Tulane Dissertation, 1970.

Received January 24, 1974. The author wishes to thank Professor Frank T. Birtel for his guidance and encouragement during the preparation of his dissertation, at Tulane University, New Orleans. Part of the material in this dissertation forms the basis for this paper. The author held a fellowship of the Niels Stensen Stichting in Amsterdam, The Netherlands, from September, 1970, until August, 1971.

New Orleans, Tulane University

AND

AMSTERDAM, UNIVERSITEIT VAN AMSTERdAM 



\section{Pacific Journal of Mathematics}

\section{Vol. 66, No. $1 \quad$ November, 1976}

Helen Elizabeth. Adams, Factorization-prime ideals in integral domains ............ Patrick Robert Ahern and Robert Bruce Schneider, The boundary behavior of Henkin's kernel.

Daniel D. Anderson, Jacob R. Matijevic and Warren Douglas Nichols, The Krull

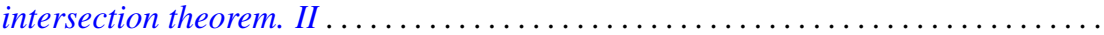

Efraim Pacillas Armendariz, On semiprime P.I.-algebras over commutative regular

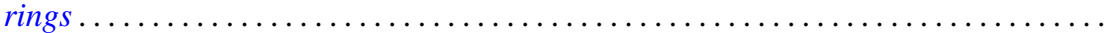

Robert H. Bird and Charles John Parry, Integral bases for bicyclic biquadratic fields

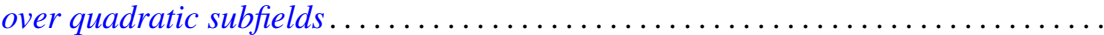

Tae Ho Choe and Young Hee Hong, Extensions of completely regular ordered

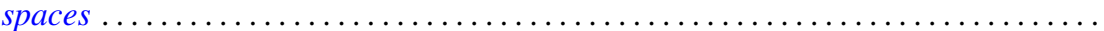

John Dauns, Generalized monoform and quasi injective modules ...............

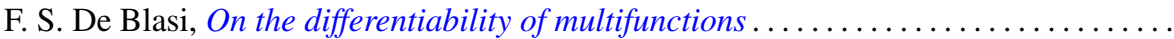

Paul M. Eakin, Jr. and Avinash Madhav Sathaye, R-endomorphisms of $R[[X]]$ are

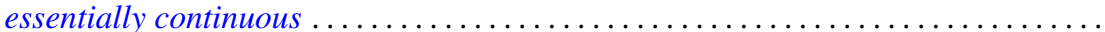

Larry Quin Eifler, Open mapping theorems for probability measures on metric

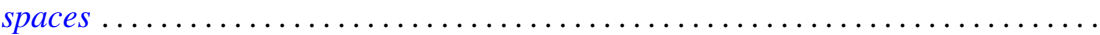

Garret J. Etgen and James Pawlowski, Oscillation criteria for second order self adjoint

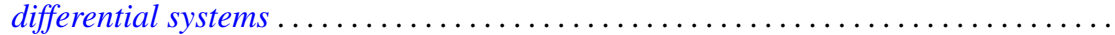

Ronald Fintushel, Local $S^{1}$ actions on 3-manifolds .

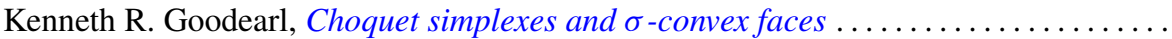

John R. Graef, Some nonoscillation criteria for higher order nonlinear differential

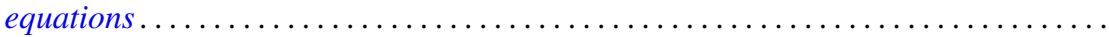

Charles Henry Heiberg, Norms of powers of absolutely convergent Fourier series: an example.

Les Andrew Karlovitz, Existence of fixed points of nonexpansive mappings in a space

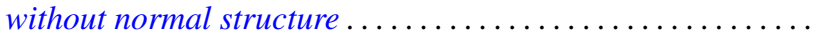

Gangaram S. Ladde, Systems of functional differential inequalities and functional

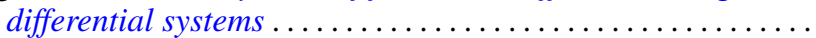

Joseph Michael Lambert, Conditions for simultaneous approximation and interpolation with norm preservation in $C[a, b]$.

Ernest Paul Lane, Insertion of a continuous function.

Robert F. Lax, Weierstrass points of products of Riemann surfaces .

Dan McCord, An estimate of the Nielsen number and an example concerning the Lefschetz fixed point theorem...

Paul Milnes and John Sydney Pym, Counterexample in the theory of continuous functions on topological groups...

Peter Johanna I. M. De Paepe, Homomorphism spaces of algebras of holomorphic functions

Judith Ann Palagallo, A representation of additive functionals on $L^{p}$-spaces,

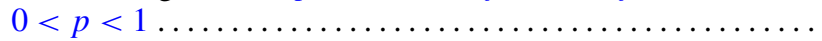

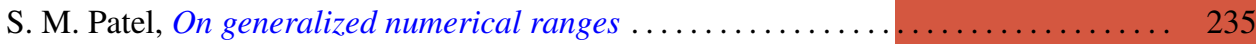

Thomas Thornton Read, A limit-point criterion for expressions with oscillatory

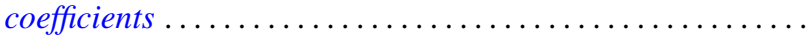

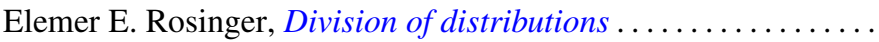

Peter S. Shoenfeld, Highly proximal and generalized almost finite

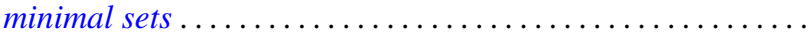

R. Sirois-Dumais and Stephen Willard, Quotient-universal sequential spaces

Robert Charles Thompson, Convex and concave functions of singular values of matrix sums....

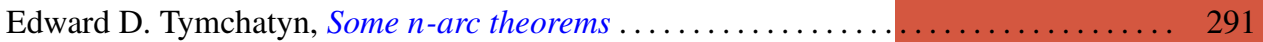

